Lal, Sohan; Lucas, Jan; Juurlink, Ben

\title{
E2MC: Entropy Encoding Based Memory Compression for GPUs
}

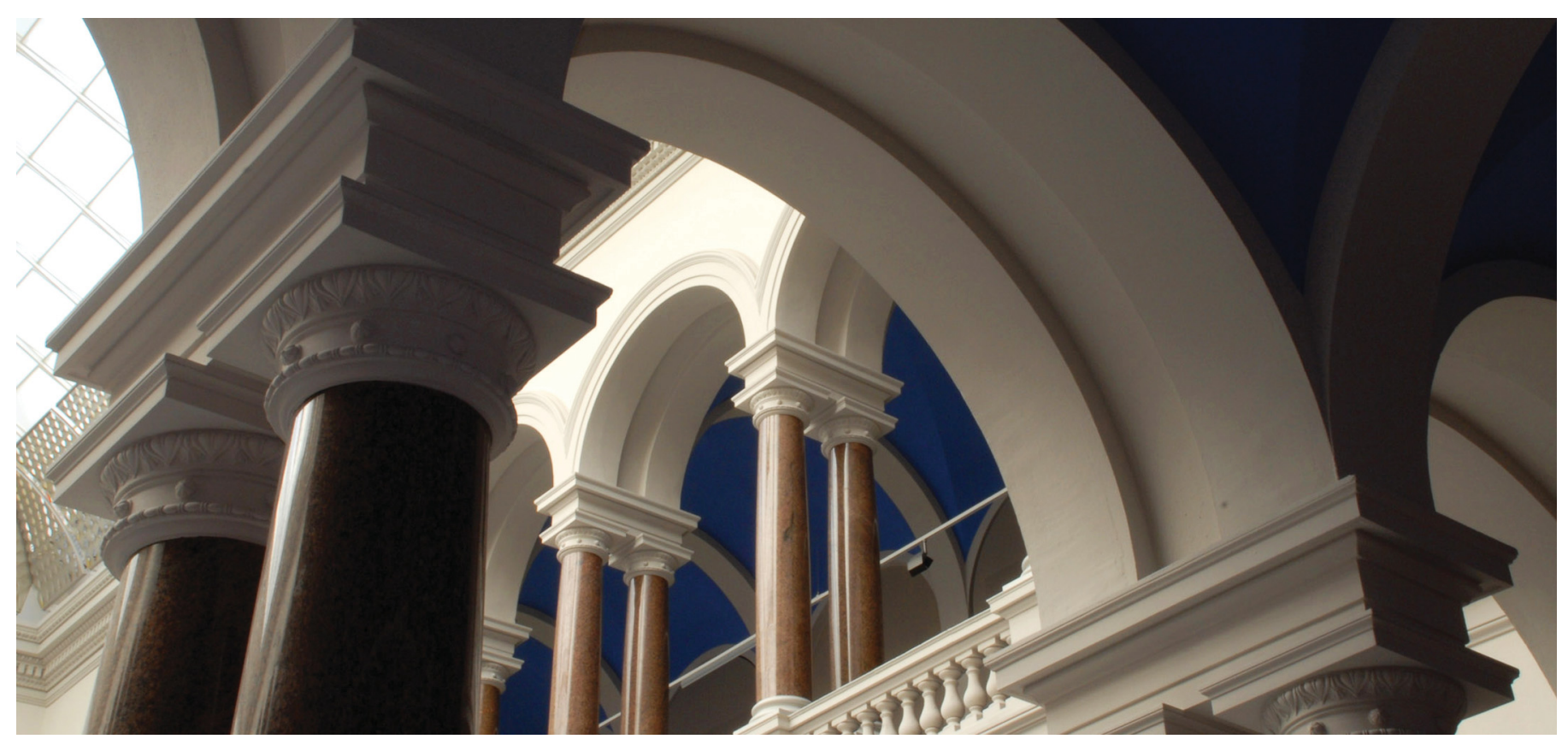

Lal, S., Lucas, J., \& Juurlink, B. (2017). E2MC: Entropy Encoding Based Memory Compression for GPUs. In 2017 IEEE International Parallel and Distributed Processing Symposium (IPDPS). IEEE.

https://doi.org/10.1109/ipdps.2017.101 


\title{
$\mathrm{E}^{2} \mathrm{MC}$ : Entropy Encoding Based Memory Compression for GPUs
}

\author{
Sohan Lal, Jan Lucas, Ben Juurlink \\ Technische Universität Berlin, Germany \\ \{ sohan.lal, j.lucas, b.juurlink\}@tu-berlin.de
}

\begin{abstract}
Modern Graphics Processing Units (GPUs) provide much higher off-chip memory bandwidth than CPUs, but many GPU applications are still limited by memory bandwidth. Unfortunately, off-chip memory bandwidth is growing slower than the number of cores and has become a performance bottleneck. Thus, optimizations of effective memory bandwidth play a significant role for scaling the performance of GPUs.

Memory compression is a promising approach for improving memory bandwidth which can translate into higher performance and energy efficiency. However, compression is not free and its challenges need to be addressed, otherwise the benefits of compression may be offset by its overhead. We propose an entropy encoding based memory compression $\left(\mathrm{E}^{2} \mathrm{MC}\right)$ technique for GPUs, which is based on the well-known Huffman encoding. We study the feasibility of entropy encoding for GPUs and show that it achieves higher compression ratios than state-of-the-art GPU compression techniques. Furthermore, we address the key challenges of probability estimation, choosing an appropriate symbol length for encoding, and decompression with low latency. The average compression ratio of $\mathrm{E}^{2} \mathrm{MC}$ is $53 \%$ higher than the state of the art. This translates into an average speedup of $20 \%$ compared to no compression and $8 \%$ higher compared to the state of the art. Energy consumption and energy-delayproduct are reduced by $13 \%$ and $27 \%$, respectively. Moreover, the compression ratio achieved by $E^{2} \mathrm{MC}$ is close to the optimal compression ratio given by Shannon's source coding theorem.
\end{abstract}

\section{INTRODUCTION}

GPUs are high throughput devices which use fine-grained multi-threading to hide the long latency of accessing off-chip memory [1]. Threads are grouped into fixed size batches called warps in NVIDIA terminology. The GPU warp scheduler chooses a new warp from a pool of ready warps if the current warp is waiting for data from memory. This is effective for hiding the memory latency and keeping the cores busy for compute-bound benchmarks. However, for memory-bound benchmarks, all warps are usually waiting for data from memory and performance is limited by off-chip memory bandwidth. Performance of memory-bound benchmarks increases when additional memory bandwidth is provided. Figure 1 shows the speedup of memory-bound benchmarks when the offchip memory bandwidth is increased by $2 \times, 4 \times$, and $8 \times$. The average speedup is close to $2 \times$, when the bandwidth is increased by $8 \times$. An obvious way to increase memory bandwidth is to increase the number of memory channels and/or their speed. However, technological challenges, cost, and other limits restrict the number of memory channels and/or their speed [2], [3]. Moreover, research has already shown that memory is a significant power consumer [4], [5], [6] and

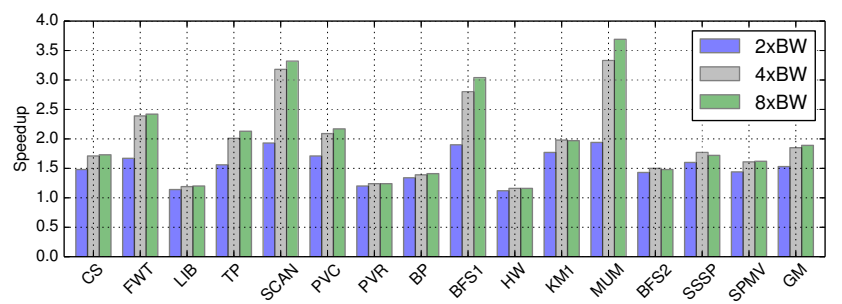

Fig. 1: Speedup with increased memory bandwidth

increasing the number of memory channels and/or frequency elevates this problem. Clearly, alternative ways to tackle the memory bandwidth problem are required.

A promising technique to increase the effective memory bandwidth is memory compression. However, compression incurs overheads such as (de)compression latency which need to be addressed, otherwise the benefits of compression could be offset by its overhead. Fortunately, GPUs are not as latency sensitive as CPUs and they can tolerate latency increases to some extent. Moreover, GPUs often use streaming workloads with large working sets that cannot fit into any reasonably sized cache. The higher throughput of GPUs and streaming workloads mean bandwidth compression techniques are even more important for GPUs than CPUs. The difference in the architectures offers new challenges which need to be tackled as well as new opportunities which can be harnessed.

Most existing memory compression techniques exploit simple patterns for compression and trade low compression ratios for low decompression latency. For example, FrequentPattern Compression (FPC) [7] replaces predefined frequent data patterns, such as consecutive zeros, with shorter fixedwidth codes. C-Pack [8] utilizes fixed static patterns and dynamic dictionaries. Base-Delta-Immediate (BDI) compression [9] exploits value similarity. While these techniques can decompress with few cycles, their compression ratio is low, typically only $1.5 \times$. All these techniques originally targeted CPUs and hence, traded low compression for lower latency.

As GPUs can hide latency to a certain extent, more aggressive entropy encoding based data compression techniques such as Huffman compression seems feasible. While entropy encoding could offer higher compression ratios, these techniques also have inherent challenges which need to be addressed. The main challenges are 1) How to estimate probability? 2) What is an appropriate symbol length for encoding? And 3) How to keep the decompression latency low? In this paper, we address these 
key challenges and propose to use Entropy Encoding based Memory Compression ( $\mathrm{E}^{2} \mathrm{MC}$ ) for GPUs.

We use both offline and online sampling to estimate probabilities and show that small online sampling results in compression comparable to offline sampling. While GPUs can hide a few tens cycles of additional latency, too many can still degrade their performance [10]. We reduce the decompression latency by decoding multiple codewords in parallel. Although parallel decoding reduces the compression ratio because additional information needs to be stored, we show that the reduction is not much as it is mostly hidden by the memory access granularity (MAG). The MAG is the minimum amount of data that is fetched from memory in response to a memory request and it is a multiple of burst length and memory bus width. For example, the MAG for GDDR5 is either 32B or 64B depending upon if the bus width is 32-bit or 64-bit respectively. Moreover, GPUs are high throughput devices and, therefore, the compressor and decompressor should also provide high throughput. We also estimate the area and power needed to meet the high throughput requirements of GPUs. In summary, we make the following contributions:

- We show that entropy encoding based memory compression is feasible for GPUs and delivers higher compression ratio and performance gain than state-of-the-art techniques.

- We address the key challenges of probability estimation, choosing a suitable symbol length for encoding, and low decompression latency by parallel decoding with negligible or small loss of compression ratio.

- We provide a detailed analysis of the effects of memory access granularity on the compression ratio.

- We analyze the high throughput requirements of GPUs and provide an estimate of area and power needed to support such high throughput.

- We further show that compression ratio achieved is close to the optimal compression ratio given by Shannon's source coding theorem.

This paper is organized as follows. Section II describes related work. In Section III we present $\mathrm{E}^{2} \mathrm{MC}$ in detail. Section IV explains the experimental setup and experimental results are presented in Section V. Finally, we draw conclusions in Section VI.

\section{RELATED WORK}

Frame buffer and texture compression have been adopted by commercial GPUs. For example, ARM Frame Buffer Compression [11] is available on Mali GPUs. AFBC is claimed to reduce the required memory bandwidth by $50 \%$ for graphics workloads. NVIDIA also uses DXT compression techniques for color compression [12]. However, these techniques only compress color and texture data and not data for GPGPU workloads. Furthermore, the micro-architectural details of most GPU compression techniques are proprietary.

Recent research has shown that compression can be used for GPGPU workloads [13], [14], [15]. Sathish et al. [13] use C-Pack [8], a dictionary based compression technique to compress data transferred through memory I/O links and show performance gain for memory-bound applications. However, they do not report compression ratios and their primary focus is to show that compression can be applied to GPUs and not how much can be compressed. Lee et al. [15] use BDI compression [9] to compress data in the register file. They observe that the computations that depend on the threadindices operate on register data that exhibit strong value similarity and there is a small arithmetic difference between two consecutive thread registers. Vijaykumar et al. [14] use underutilized resources to create assist warps that can be used to accelerate the work of regular warps. These assist warps are scheduled and executed in hardware. They use assist warps to compress cache block. In contrast to this, our compression technique is hardware based and is completely transparent to the warps. The assist warps may compete with the regular warps and can potentially affect the performance. Compared to FPC, C-PACK and BDI, we use entropy encoding based compression which results in higher compression ratio.

Huffman-based compression has been used for cache compression for CPUs [16], but to the best of our knowledge no work has used Huffman-based memory compression for GPUs. In this work Arelakis and Stenstrom [16] sample the $1 \mathrm{~K}$ most frequent values and use 32-bit symbol length for compression. In contrast to CPUs, GPUs have been optimized for FP operations and 32-bit granularity does not work well for GPUs. We show higher compression ratio and performance gain for 16-bit symbols instead of 32-bit in $\mathrm{E}^{2} \mathrm{MC}$.

\section{HufFman-BASEd MEMory BANDWidth COMPRESSION}

First, we provide an overview of a system with entropy encoding based memory compression ( $E^{2} \mathrm{MC}$ ) for GPUs and its key challenges and then in the subsequent sections address these challenges in detail.

\section{A. Overview}

Figure 2a shows an overview of a system with main components of the $\mathrm{E}^{2} \mathrm{MC}$ technique. The memory controller (MC) is modified to integrate the compressor, decompressor and metadata cache (MDC). Depending on the memory request type, either it needs to pass through the compressor or it can directly access the memory. A memory write request passes through the compressor while a read request can bypass the compressor. The MDC is updated with the size of the compressed block and finally the compressed block is written to memory. A memory read request first accesses the MDC to determine the memory request size and then fetches that much data from memory. (De)compression takes place in the $\mathrm{MC}$ and is completely transparent to the $\mathrm{L} 2$ cache and the streaming multiprocessors. The compressed data is stored in the DRAM. However, the goal is not to increase the effective capacity of the DRAM but to increase the effective off-chip memory bandwidth similar to [13]. Hence, a compressed block is still allocated the same size in DRAM, although it may require less space. Like [14], $\mathrm{E}^{2} \mathrm{MC}$ requires compression when the data is transferred from CPU to GPU to initialize the MDC. As the (de)compressors 


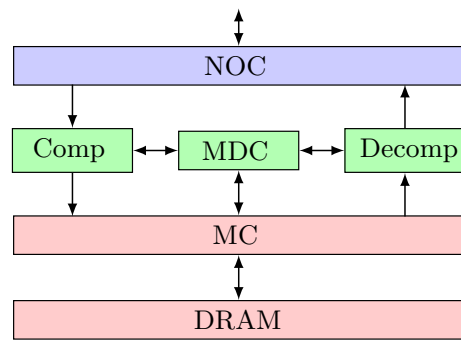

(a) System overview

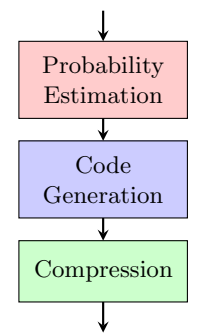

(b) Phases of entropy encoding
Fig. 2: System overview and compression phases

are integrated in the MC, data is compressed while transferring from CPU to GPU and vice versa.

\section{B. Huffman Compression and Key Challenges}

Huffman compression is based on the evidence that not all symbols have same probability. Instead of using fixed-length codes, Huffman compression uses variable-length codes based on the relative frequency of different symbols. A fixed-length code assumes equal probability for all symbols and hence assigns same length codes to all symbols. In contrast to fixedlength codes, Huffman compression is based on the principle to use fewer bits to represent frequent symbols and more bits to represent infrequent symbols. In general, compression techniques based on variable-length codes can provide high compression ratio, but they also have high overhead in terms of latency, area, and power [16]. Moreover, to achieve high compression and performance, certain key challenges should be addressed.

The first challenge is to find an appropriate symbol length (SL). The choice of SL is a very important factor as it affects compression ratio, decompression latency and hardware cost. We evaluate the tradeoffs of using different SLs (4, 8, 16, 32bit). The second challenge is accurate probability estimation. We perform both offline and online sampling of data to estimate the probability of symbols and show that it is possible to achieve compression ratio comparable to offline sampling with small online sampling. The third important factor is low decompression latency, which affects the performance gain. We reduce the decompression latency by decoding in parallel. In the following sections, we discuss these challenges in detail.

1) Choice of Symbol Length: We encode with different SLs of $4,8,16$, and 32-bit and evaluate their tradeoffs to make sure that not only the compression ratio but other aspects are also compared. Figure 3 shows the compression ratio for different SLs (Benchmarks are described in Section IV). It can be seen that 16-bit encoding yields the highest compression ratio for GPUs. This result is in contrast to [16] where it was shown that 16 and 32-bit encodings yield almost same compression ratio for CPUs. The next highest compression ratio is provided by 8-bit symbols. For some benchmarks (TP, MUM, SPMV), 8-bit encoding offers the highest compression ratio. GPUs often operate on FP values, but INT values are also not uncommon. Most of the benchmarks in MARS [17] and Lonestar [18] suites are INT. Often smaller symbols are more effective for

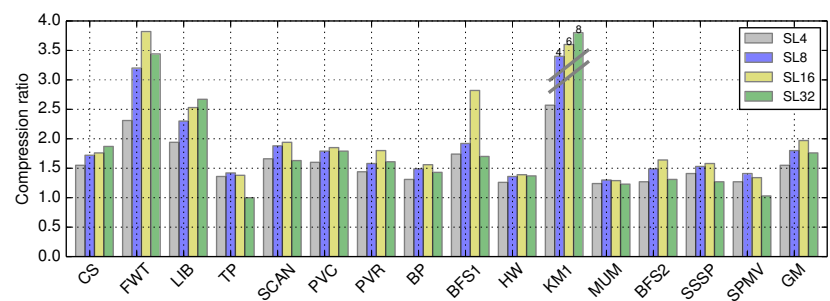

Fig. 3: Compression ratio for different symbol lengths

FP, while longer symbols are more effective for INT and on average 16-bit symbols provide good tradeoff for both.

2) Probability Estimation: Figure $2 \mathrm{~b}$ shows the different phases of an entropy encoding based compression technique. In the probability estimation phase, frequencies of the different symbols are collected. Based on frequencies, variable-length codes are assigned to different symbols. In the final phase, compression takes place. Accurate probability estimation is one of the key components of entropy based compression. Therefore, in our proposed $\mathrm{E}^{2} \mathrm{MC}$ technique we use both offline probability estimation where we estimate the probability of symbols offline, and online probability estimation where we sample the probability of symbols during runtime.

a) Offline Probability Estimation: We simulate all benchmarks and store their load and store data in a database. Then we profile the database offline to find probability of symbols. Offline probability estimate is the best estimate we can have. In Section $\mathrm{V}$ it is shown that offline probability yields the highest compression ratio. However, offline probability can only be used if approximate entropy characteristics of a data stream are known in advance.

b) Online Probability Estimation: One of the drawbacks of entropy based compression techniques is that they may require online sampling to estimate the frequency of symbols if entropy characteristics are not known in advance. The sampling phase is an additional overhead as during sampling no compression is performed. Fortunately, our experiments show that it is possible to achieve compression ratio comparable to offline probability with a very short online sampling phase at the start of the benchmarks.

During sampling phase every memory request is monitored at the memory controller to record unique values and their count. We use a value frequency table (VFT) similar to [16] to store them. For 4 and 8-bit SLs, we store all values as there are only 16 and 256 possible values. However, for SLs of 16 and 32-bit we only store the most frequent values (MFVs) and use them to generate codewords as the total number of values is very large and storing all of them is not practical.

There are two key decisions to make regarding online sampling. First, what is the suitable number of MFVs? More MFVs may help to encode better for some benchmarks at the cost of increased hardware. Figure 4a shows the compression ratio for different numbers of $\mathrm{MFVs}$ relative to $1 \mathrm{~K}$ MFVs. It shows that the compression ratio does not increase much with the number of MFVs, only $1 \%$ on average. Only for some benchmarks (FWT, TP, SCAN, SPMV) more MFV gives 


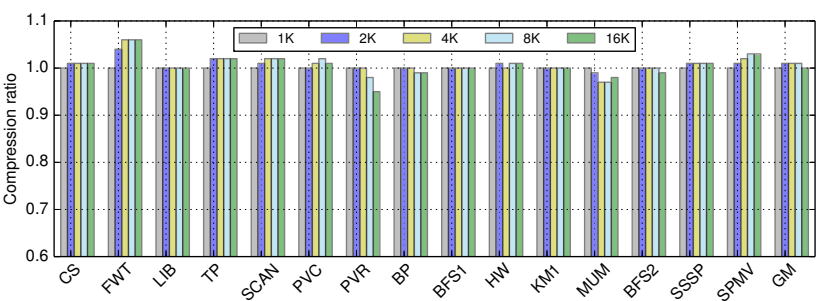

(a) Compression ratio with different number of MFVs

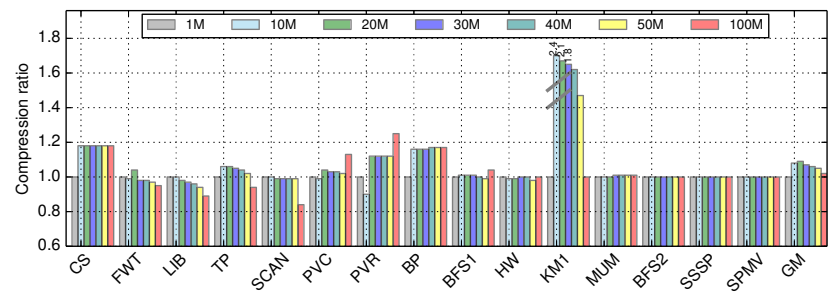

(b) Compression ratio with different sampling durations

Fig. 4: Online sampling decisions

slightly higher compression ratio, FWT being the highest gainer (6\%). Hence, we choose $1 \mathrm{~K}$ MFVs to construct encoding. In some cases (PVR, MUM), the compression ratio can even decrease with the increase in MFVs as the length of the prefix which is attached to each uncompressed symbol can increase. For example, for the MUM benchmark, the prefix is 3 bits long for $2 \mathrm{~K} \mathrm{MFVs}$ and 4 bits long for $4 \mathrm{~K}$ MFVs. Thus, the compression ratio is lower for $4 \mathrm{~K}$ MFVs compared to $2 \mathrm{~K}$ MFVs.Please refer to Section III-B2c for more details.

The second decision is: what is the best sampling duration? Figure $4 \mathrm{~b}$ shows the compression ratio for sampling durations of $1 \mathrm{M}, 10 \mathrm{M}, 20 \mathrm{M}, 30 \mathrm{M}, 40 \mathrm{M}, 50 \mathrm{M}$ and $100 \mathrm{M}$ instructions relative to sampling duration of $1 \mathrm{M}$ instructions. It shows that sampling for $20 \mathrm{M}$ instructions yields the highest compression ratio. Hence, we choose 20M instructions for online sampling. The longer sampling duration can improve probability estimation, however, there is a trade-off between compression ratio and improved probability estimation as no compression is done during sampling duration. Thus, we notice low compression ratio for sampling durations longer than $20 \mathrm{M}$ instructions.

c) Codeword Generation: After the sampling phase, code generation takes place. Instead of classic Huffman coding, canonical Huffman coding [19] is used because it is fast to decode as the codeword length is enough to decode a codeword. In canonical Huffman coding, codewords of the same length are consecutive binary numbers. To generate canonical Huffman codewords (CHCs), first classic codewords are generated by building a Huffman tree using a minimum heap data structure as described in [20] and then symbols are sorted according to their code lengths. The first symbol is assigned a codeword of all zeros of length equal to the original SL. The next codeword is just the consecutive binary number if the SL is the same. When a longer codeword is encountered, the last canonical codeword is incremented by one and left shifted until its length is equal to the original SL. The procedure is repeated for all

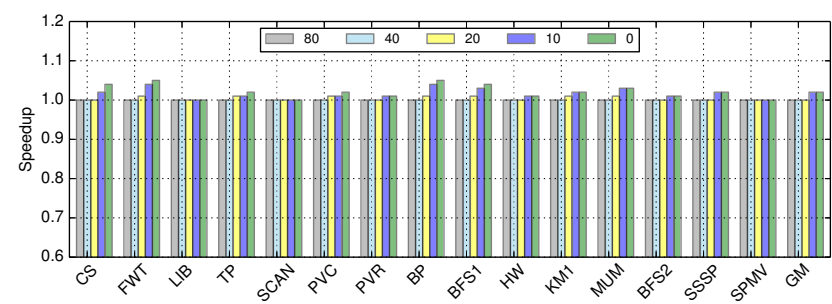

(a) Effect of compression latency on speedup

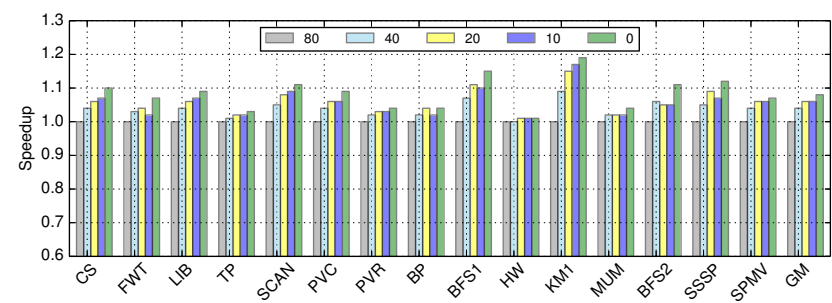

(b) Effect of decompression latency on speedup

Fig. 5: Effect of latency

symbols. For example, assume we have three symbols $(\mathrm{A}=$ $(11)_{2}, \mathrm{~B}=(0)_{2}, \mathrm{C}=(101)_{2}$ ) with classic Huffman codewords. To convert them to canonical, we first sort symbols according to code length $\left(\mathrm{B}=(0)_{2}, \mathrm{~A}=(11)_{2}, \mathrm{C}=(101)_{2}\right)$ and then the first symbol (B) is assigned code $(0)_{2}$. The $\mathrm{CHC}$ for the next symbol $\mathrm{A}$ is $(10)_{2}$ which is obtained by incrementing the last codeword by one and then left shifting to match the original code length of A. Similarly, CHC for C is $(110)_{2}$.

To ensure that unnecessarily long codewords are not generated, we assign a minimum probability to each symbol such that no codeword is longer than predetermined maximum length. Our experiments show that there are only few symbols which have codewords longer than 20-bit for SL 16 and 32-bit. Hence, we adjust the frequency so that no symbol is assigned a code longer than 20-bit. Similarly, for SL 4 and 8-bit we fix the maximum codeword length to 8 and 16-bit, respectively. Finally, all symbols that are not in MFVs are assigned a single codeword based on their combined frequency and this codeword is attached as a prefix to store all such symbols uncompressed. Since we only need probability estimation and code generation in the beginning, both of these steps can be done in software.

3) Low Decompression Latency: As already explained, GPUs are high throughput devices and less sensitive to latency than CPUs. However, large latency increases can also affect GPU performance. Figure $5 \mathrm{a}$ and $5 \mathrm{~b}$ shows the speedup when compression and decompression latency is decreased from 80 cycles to 0 cycles, respectively. It can be seen that there is a small speedup (geometric mean of $1 \%$ ) when the compression latency is decreased to 0 cycles. However, there is a significant speedup (geometric mean of $9 \%$ ) when the decompression latency is decreased to 0 cycles. The speedup is more sensitive to decompression latency because warps have to stall for loads from memory, while stores can be done without stalling. The results clearly show the importance of low decompression latency. Thus, we perform parallel decoding to decrease the 


\begin{tabular}{ll||ll}
\hline $\mathrm{DDR}_{x}$ & $\mathrm{BL}$ & $\mathrm{DDR}_{x}$ & $\mathrm{BL}$ \\
\hline $\mathrm{DDR} 1$ & 2 & DDR3/4 & 8 \\
\hline DDR2 & 4 & GDDR5 & 8 \\
\hline
\end{tabular} \begin{tabular}{lll||lll} 
& $\mathrm{CR}$ & $\mathrm{BF}$ & $\mathrm{CR}$ & $\mathrm{BF}$ \\
\hline $1.00-1.33$ & $128 \mathrm{~B}$ & $2.00-3.99$ & $64 \mathrm{~B}$ \\
\hline $1.33-1.99$ & $96 \mathrm{~B}$ & $\geq 4.00$ & $32 \mathrm{~B}$ \\
\hline
\end{tabular}

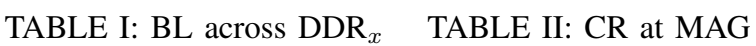

decompression latency as explained in Section III-F1.

\section{Memory Access Granularity and Compression}

Memory access granularity (MAG) is the number of bytes fetched from memory at a time and it is a product of the burst length (BL) and bus width. The BL is decided by DRAM technology, and it directly determines the MAG. Table I shows the $\mathrm{BL}$ for different generations of $\mathrm{DDR}_{x}$. The $\mathrm{BL}$ has increased over the generations to support high data transfer rates.

The MAG is an important factor for memory compression as it affects the minimum amount of data that can be fetched from memory. Assuming 32-bit bus width for DDR4, the MAG of DDR4 is 32B. Since it is only possible to fetch in multiples of the MAG, for a compression ratio (CR) that is not an exact multiple of the MAG, more data needs to be fetched than what is actually needed. For example, for a block that is compressed from 128B to 65B (CR of 1.97), the actual amount of fetched data is $96 \mathrm{~B}(3 \times 32 \mathrm{~B})$. Thus, while the $\mathrm{CR}$ looks very close to 2 , the effective CR is only 1.33 . Therefore, effective compression and performance gain could be significantly less than what it appears at first due to MAG restrictions. Table II shows range of $\mathrm{CR}$ and minimum number of bytes fetched (BF) assuming 32B MAG. We assume the MAG is 32B for this study.

\section{Compression Overhead: Metadata Cache}

To save memory bandwidth as a result of compression, we need to only fetch the compressed 32B bursts from a DRAM. Therefore, the memory controller needs to know how many bursts to fetch for every memory block. For GDDR5, the number of bursts varies from 1 to 4 . Similar to previous work [13], [14], we store 2 bits for every memory block as metadata. For a 32-bit, 4GB DRAM with block size of 128B, we need $8 \mathrm{MB}$ of DRAM for storing the metadata. However, we cannot afford to access the metadata first from DRAM and then issue a memory request for the required number of bursts. This requires two accesses to DRAM and defeats the purpose of compression to save memory bandwidth. Therefore, like previous work [13], [14], we cache the most recently used metadata in a cache. We use a small $8 \mathrm{~KB} 4$-way set associate cache to store the metadata. The 2 bits stored in the metadata are also used to determine if the block is stored (un)compressed. The value $(11)_{2}$ means the block is stored uncompressed.

\section{E. Huffman Compressor}

Figure 6 shows an overview of the Huffman compressor. It can be implemented as a small lookup table (c-LUT) that stores codewords (CWs) and their code lengths (CLs) as shown in Figure 6a. The maximum number of CWs is $2^{N}$, where $N$ is the SL. As the maximum number of CWs is only 16 and 256 for SLs 4 and 8-bit, we store all CWs in a c-LUT and index it directly using symbol bits. However, for SLs 16 and 32-bit such a lookup table is not practical and instead we store

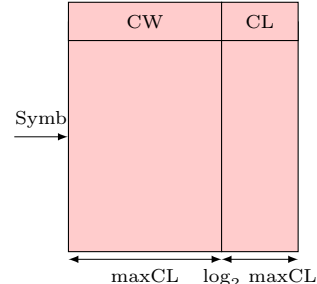

(a) c-LUT

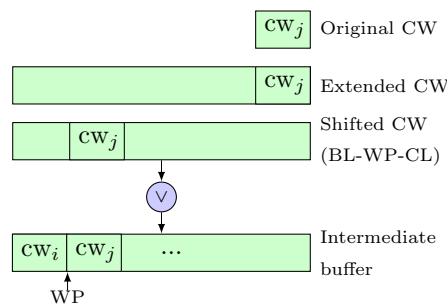

(b) Placing codewords together
Fig. 6: Huffman compressor

$1 \mathrm{~K}$ MFVs as discussed in Section III-B2b. We use an 8-way set associative cache to implement c-LUT for $1 \mathrm{~K}$ MFVs. The cache is indexed by lower 7-bit of a symbol.

For SLs 4 and 8-bit, we build different Huffman trees corresponding to different symbols in a word and thus, also use multiple c-LUTs, one corresponding to each tree. For example, for SL 8-bit, we use $4 \mathrm{c}$-LUTs for the four different symbols in a word. We assume a word size of 32-bit for our study.

Once we obtain a CW from c-LUT, we need to place it together with other CWs. Figure $6 \mathrm{~b}$ shows how the CWs are placed together. We use an intermediate buffer of $2 \times \operatorname{maxCL}$ as buffer length (BL), where maxCL is the maximum code length. To place a $\mathrm{CW}$ at its right position, first the $\mathrm{CW}$ is extended to match BL and then the extended $\mathrm{CW}$ is left shifted by $B L-W P-C L$ using a barrel shifter, where WP is the current write position in the buffer. Finally, the shifted $\mathrm{CW}$ is bitwise ORed with the intermediate buffer and WP is incremented by CL. When WP $\geq \operatorname{maxCL}$, compressed data, equal to maxCL, is moved from the intermediate buffer to the final buffer. The intermediate buffer is left shifted by maxCL and WP is decremented by maxCL. Our RTL synthesis shows that placing the CWs together takes more time than getting $\mathrm{CW}$ and CL from c-LUT. The sum of the lengths of all CWs of a block determines if a block is stored (un)compressed. When the sum is $\leq 96 \mathrm{~B}$, a block is stored compressed, otherwise uncompressed. Please refer to Section III-C to understand the reason to choose compressed size $\leq 96 \mathrm{~B}$ to decide if a block is stored (un)compressed.

\section{F. Huffman Decompressor}

Figure 7 shows an overview of the Huffman decompressor. Our design is based on the Huffman decompressor as proposed in [16], which mainly consists of a barrel shifter, comparators, and a priority encoder to find the CW and CL. We use a buffer of length $2 \times$ maxCL to store part of the compressed block. We use buffer length of $2 \times$ maxCL instead of maxCL as in [16] for two reasons. First, we can continue decoding without shifting data every cycle from the compressed block to the buffer. Second, it helps to define a fixed-width interface (maxCL in this case) to input compressed data instead of every cycle shifting a different number of bits, equal to the matched CL. A pointer (bufPos) which initially points to the end of the buffer is used to track the filled size of the buffer. To find a $\mathrm{CW}$, comparison is done in parallel between all potential CWs and the First Codewords (FCWs) of all lengths. The FCWs 


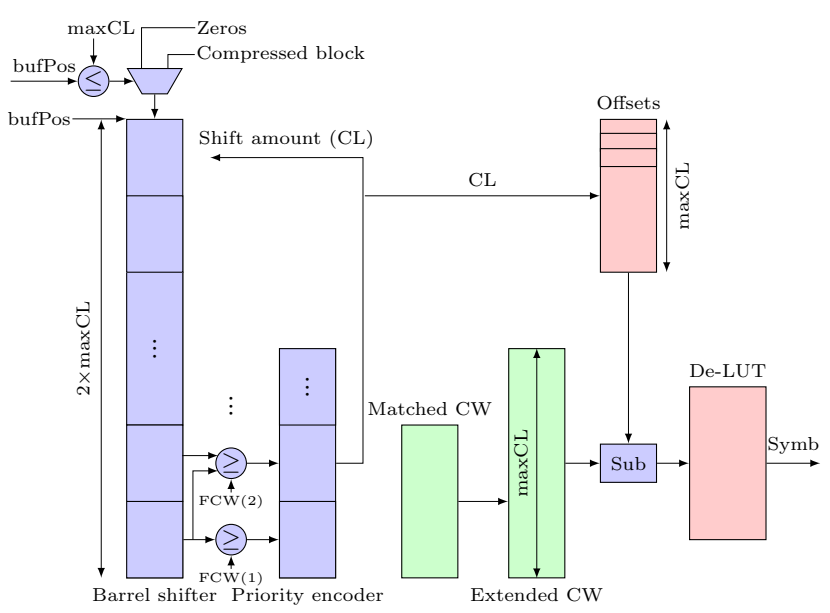

Fig. 7: Huffman decompressor [16]

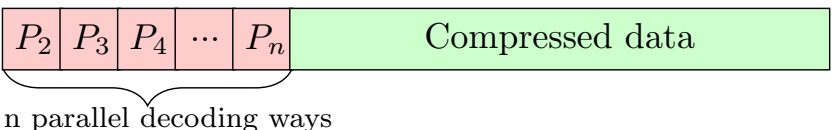

$\mathrm{n}$ parallel decoding ways

Fig. 8: Structure of a compressed block

of all lengths are stored in a table. A priority encoder selects the first $\mathrm{CW}$ which is $\geq \mathrm{FCW}$ of length $l$ and $\leq \mathrm{FCW}$ of length $l+1$. The selected $\mathrm{CW}$ is extended by padding zeros to match the maxCL. An offset which depends on the CL and is calculated during code generation is subtracted from $\mathrm{CW}$ to obtain the index for the De-LUT. The barrel shifter shifts the buffer by CL and the bufPos is decremented by CL. When the bufPos $\leq$ maxCL, the remaining compressed data of length maxCL is shifted into the buffer from the compressed block and the bufPos is incremented by maxCL.

Although symbols are stored in consecutive locations in the De-LUT, canonical CWs of different CLs are not consecutive binary numbers. Therefore, the De-LUT cannot be indexed directly using the $\mathrm{CW}$ to obtain the decoded symbol. We need to subtract an offset from the $\mathrm{CW}$ to find the index. These offsets are calculated during code generation. For example, assume we have three symbols with canonical Huffman codewords (CHCs) $\left(\mathrm{B}=(0)_{2}, \mathrm{~A}=(10)_{2}, \mathrm{C}=(110)_{2}\right)$. The symbol $\mathrm{B}$ will be stored at index 0 in the De-LUT, so it has offset 0 . However, A will be stored at index 1 , so it has offset $1\left((10)_{2^{-}}\right.$ $\left.(01)_{2}\right)$. Similarly, $\mathrm{C}$ will be stored at index 2 and it has offset 4 $\left((110)_{2}-(010)_{2}\right)$. The maximum number of offsets is equal to maximum number of CWs of different lengths. Both offset and FCW tables are read and writable so that they can be changed for different benchmarks. We use multiple decompressor units for different symbols in a word for SLs 4 and 8-bit.

1) Parallel Decoding and Memory Access Granularity: We need to decode in parallel to reduce the decompression latency. Unfortunately, Huffman decoding is serial as the start of the next $\mathrm{CW}$ is only known once the previous $\mathrm{CW}$ has been found. Serial decoding requires high latency which can limit the performance gain. One way to parallelize Huffman decoding is to explicitly store pointers to $\mathrm{CWs}$ where we want

\begin{tabular}{|c|c|c|c|c|c|c|c|c|}
\hline & \multicolumn{4}{|c|}{ Compressor } & \multicolumn{4}{|c|}{ Decompresor } \\
\hline $\begin{array}{l}\text { SL } \\
\text { (Bits) }\end{array}$ & $\begin{array}{l}\text { Freq } \\
(\mathrm{GHz})\end{array}$ & $\begin{array}{l}\mathrm{BW} \\
(\mathrm{GB} / \mathrm{s})\end{array}$ & $\begin{array}{l}\text { Area } \\
\left(m^{2}\right)\end{array}$ & $\begin{array}{l}\text { Power } \\
(\mathrm{mW})\end{array}$ & $\begin{array}{l}\text { Freq } \\
(\mathrm{GHz})\end{array}$ & $\begin{array}{l}\mathrm{BW} \\
(\mathrm{GB} / \mathrm{s})\end{array}$ & $\begin{array}{l}\text { Area } \\
\left(\mathrm{mm}^{2}\right)\end{array}$ & $\begin{array}{l}\text { Power } \\
(\mathrm{mW})\end{array}$ \\
\hline 4 & 1.67 & 0.84 & 0.02 & 1.43 & 1.11 & 0.56 & 0.01 & 5.12 \\
\hline 8 & 1.54 & 1.54 & 0.08 & 4.01 & 0.91 & 0.91 & 0.04 & 11.91 \\
\hline 16 & 1.43 & 2.86 & 0.11 & 5.47 & 0.80 & 1.60 & 0.07 & 11.89 \\
\hline 32 & 1.43 & 5.72 & 0.17 & 9.34 & 0.80 & 3.20 & 0.12 & 14.30 \\
\hline
\end{tabular}

TABLE III: Frequency, bandwidth, area, and power of a single unit of compressor and decompressor

\begin{tabular}{l||lll||lll||ll}
\hline \multicolumn{1}{l||}{} & \multicolumn{3}{c||}{ Compressor } & \multicolumn{3}{c||}{ Decompresor } & \multicolumn{2}{|c}{ GTX580 } \\
\hline $\begin{array}{l}\text { SL } \\
(\text { Bits })\end{array}$ & $\begin{array}{l}\text { Units } \\
(\#)\end{array}$ & $\begin{array}{l}\text { Area } \\
\left(\mathrm{mm}^{2}\right)\end{array}$ & $\begin{array}{l}\text { Power } \\
(\mathrm{W})\end{array}$ & $\begin{array}{l}\text { Units } \\
(\#)\end{array}$ & $\begin{array}{l}\text { Area } \\
\left(\mathrm{mm}^{2}\right)\end{array}$ & $\begin{array}{l}\text { Power } \\
(\mathrm{W})\end{array}$ & $\begin{array}{l}\text { Area } \\
(\%)\end{array}$ & $\begin{array}{l}\text { Power } \\
(\%)\end{array}$ \\
\hline 4 & 464 & 7.9 & 0.7 & 692 & 10.3 & 3.6 & 3.4 & 1.7 \\
\hline 8 & 252 & 20.3 & 1.0 & 424 & 18.1 & 5.0 & 7.3 & 2.5 \\
\hline 16 & 136 & 14.6 & 0.7 & 240 & 16.4 & 2.8 & 5.8 & 1.5 \\
\hline 32 & 68 & 11.5 & 0.6 & 120 & 14.3 & 1.7 & 4.9 & 0.9 \\
\hline
\end{tabular}

TABLE IV: \#units, area, and power to support $4 \times 192.4 \mathrm{~GB} / \mathrm{s}$

to start decoding in parallel in the compressed block itself. The number of pointers depends on the number of required parallel decoding ways (PDWs).

Figure 8 shows the structure of a compressed block. It consists of $n-1$ pointers $\left(P_{2}, P_{3}, \ldots, P_{n}\right)$ for $n$ PDWs, and the compressed data. Each pointer consists of $N$ bits where $2^{N}$ is the block size in bytes. For example, for a 128B block, 7-bit are needed for each PDW. The starting codewords for parallel decoding are byte-aligned while compressing them. These pointers are overhead and hence will reduce compression ratio. However, the effective loss in compression ratio is usually much lower due to the aforementioned memory access granularity (MAG). Most blocks are compressed to a size that allows adding extra bits for parallel decoding without reducing their compression ratio at the MAG. Our experiments show that even with 4 PDWs where we store 21 extra bits $(3 * 7)$ in the compressed block, there is either no or small loss in compression ratio. We analyze the loss in compression and the number of PDWs needed in Section V-A1 and V-C.

\section{G. High Throughput Requirements}

To gain from memory compression, the throughput (bytes (de)compressed per second) of the compressor and decompressor should match the full compressed memory bandwidth (BW). Suppose we can obtain a maximum compression of $4 \times$, then the compressor and decompressor throughput has to be 4 times the GPU BW to fully utilize the compressed BW. Unfortunately, a single (de)compressor unit cannot meet such high throughput.

Table III shows the frequency, BW, area, and power of a single compressor and decompressor unit as reported by the Synopsis design compiler for different SLs. The BW of NVIDIA GTX580 is $192.4 \mathrm{~GB} / \mathrm{s}$ (32.1 GB/s per memory controller). However, the combined throughput of the compressor and decompresor for any SL is far less than $4 \times 32.1 \mathrm{~GB} / \mathrm{s}$. For example, the combined throughput of the SL 16-bit is only $4.46 \mathrm{~GB} / \mathrm{s}$. Clearly, a single (de)compressor unit is not enough and we need multiple units.

Table IV shows the total number of compressor and decompressor units needed to support $4 \times 192.4 \mathrm{~GB} / \mathrm{s}$ and the 


\begin{tabular}{cc||cc}
\hline \#SMs & 16 & L1 \$ size/SM & $16 \mathrm{~KB}$ \\
\hline SM freq (MHz) & 822 & L2 \$ size & $768 \mathrm{~KB}$ \\
\hline Max \#Threads per SM & 1536 & \# Memory controllers & 6 \\
\hline Max \#CTA per SM & 8 & Memory type & GDDR5 \\
\hline Max CTA size & 512 & Memory clock & $1002 \mathrm{MHz}$ \\
\hline \#FUs per SM & 32 & Memory bandwidth & $192.4 \mathrm{~GB} / \mathrm{s}$ \\
\hline \#Registers/SM & $32 \mathrm{~K}$ & Burst length & 8 \\
\hline Shared memory/SM & 48KB & Bus width & $32-$ bits \\
\hline
\end{tabular}

TABLE V: Baseline simulator configuration

\begin{tabular}{lllllll}
\hline & BDI & FPC & $E^{2} \mathrm{MC} 4$ & E $^{2} \mathrm{MC} 8$ & E $^{2} \mathrm{MC} 16$ & $\mathrm{E}^{2} \mathrm{MC} 32$ \\
\hline Compressor latency & 1 & 6 & 154 & 84 & 46 & 24 \\
\hline Decompressor latency & 1 & 10 & 233 & 143 & 82 & 42 \\
\hline
\end{tabular}

TABLE VI: Compressor and decompressor latency in cycles

corresponding area and power. The $\mathrm{n}$ parallel decoding ways (n PDWs) utilize $\mathrm{n}$ decompressors from these total numbers of units to decode a single block in parallel. This only decreases decompression latency and does not add up to the total number of required units. Thus, no further multiplication of the numbers shown in Table IV is required for $\mathrm{n}$ PDWs.

Table IV also shows the total area and power needed as percentage of the area and peak power of the GTX580. A single (de)compressor unit requires less area and power for smaller SLs. However, the total area and power needed to support the GTX580 BW is much higher for smaller SLs as more units are required to meet the BW. We have smaller area and power for SL 4-bit because the c-LUT and De-LUT have very small number of entries (16). In general, the area numbers are likely higher than expected because the memory design library does not have exact memory designs needed to design (de)compressor and we have to combine smaller designs to get the required size. We believe that a custom design will be denser and will need less area. We found that none of the related work discussed throughput requirements of GPUs.

\section{ExPERIMENTAL Methodology}

In this section, we describe our experimental methodology.

\section{A. Simulator}

We use gpgpu-sim v3.2.1 [21] and modify it to integrate $\mathrm{BDI}, \mathrm{FPC}$ and $\mathrm{E}^{2} \mathrm{MC}$. We configure gpgpu-sim to simulate a GPU similar to NVIDIA's GF110 on the GTX580 card. The baseline simulator configuration is summarized in Table $\mathrm{V}$.

Table VI shows the (de)compressor latencies used to evaluate BDI, FPC, and $\mathrm{E}^{2} \mathrm{MC}$. For $\mathrm{E}^{2} \mathrm{MC}$, we evaluate four designs of SLs 4, 8, 16, and 32-bit denoted by $\mathrm{E}^{2} \mathrm{MC} 4, \mathrm{E}^{2} \mathrm{MC} 8, \mathrm{E}^{2} \mathrm{MC} 16$, and $\mathrm{E}^{2} \mathrm{MC} 32$, respectively. The latencies of the BDI and FPC are obtained from their published papers [9] and [7]. For $\mathrm{E}^{2} \mathrm{MC}$, we wrote RTL for the compressor and decompressor designs and then synthesized RTL using Synopsis design compiler version K-2015.06-SP4 at 32nm technology node to accurately estimate the frequency, area, and power. The compressor is pipelined using two stages. The first stage fetches the CW and CL from the c-LUT, while the second stage combines CWs together. We find that the critical path delay is in the second stage of the compressor. The decompressor is pipelined using three stages. The first stage finds a $\mathrm{CW}$, the second stage calculates the index for the De-LUT using CW and

\begin{tabular}{cccc}
\hline Name & Abbrev. & Data Type & Origin \\
\hline convolSeparable & CS & Single-precision FP & CUDA SDK \\
\hline fastWalshTrans & FWT & Single-precision FP & CUDA SDK \\
\hline libor & LIB & Single-precision FP & CUDA SDK \\
\hline transpose & TP & Single-precision FP & CUDA SDK \\
\hline scan & SCAN & INT & CUDA SDK \\
\hline PageViewCount & PVC & INT & MARS \\
\hline PageViewRank & PVR & INT & MARS \\
\hline backprop & BP & Single-precision FP & Rodinia \\
\hline bfs & BFS1 & INT & Rodinia \\
\hline heartwall & HW & Mixed & Rodinia \\
\hline kmeans & KM1 & Mixed & Rodinia \\
\hline mummergpu & MUM & INT & Rodinia \\
\hline bfs & BFS2 & INT & Lonestar \\
\hline sssp & SSSP & INT & Lonestar \\
\hline spmv & SPMV & Mixed & SHOC \\
\hline
\end{tabular}

TABLE VII: Benchmarks used for experimental evaluation

offsets, and the De-LUT is accessed in the third stage to get the decoded symbol. We find that the critical path delay is in the first stage of the decompressor. In $\mathrm{E}^{2} \mathrm{MC}$, one symbol can be (de)compressed in a single cycle of frequency listed in Table III. The frequency is calculated using critical path delay. However, the DRAM frequency is $1002 \mathrm{MHz}$ for GTX580. We scale the (de)compressor frequency and then count the number of cycles needed to (de)compress a memory block of size 128B. Furthermore, for each PDW, we assume the decompressor latency is decreased by the same factor.

For estimating the energy consumption of different benchmarks, GPUSimPow [5] is modified to integrate the power model of the compressor and decompressor. The power numbers obtained by RTL synthesis are used to derive the power model for the compressor and decompressor.

\section{B. Benchmarks}

Table VII shows the benchmarks used for evaluation. We include benchmarks from the popular CUDA SDK [22], Rodinia [23], Mars [17], Lonestar [18], and SHOC [24]. A benchmark can belong to single-precision FP, INT, or mixed category depending upon its data types. We modified the inputs of SCAN and FWT benchmarks as the original inputs were random which are not suitable for any compression technique. We use SCAN for stream compaction which is an important application of SCAN and FWT to transform Walsh functions.

\section{EXPERIMENTAL RESUlTS}

To evaluate the effectiveness of $\mathrm{E}^{2} \mathrm{MC}$, we compare compression ratio (CR) and performance of $\mathrm{E}^{2} \mathrm{MC}$ for SLs 4, 8, 16, and 32-bit with BDI and FPC. We provide two kinds of compression ratios, raw CR and CR at memory access granularity (MAG). The raw $\mathrm{CR}$ is the ratio of the total uncompressed size to total compressed size. For CR at MAG, the total compressed size is calculated by scaling up the compressed size of each block to the nearest multiple of MAG, and then adding all the scaled block sizes.

First, we present CR results using offline and online probability estimation and discuss $\mathrm{CR}$ and parallel decoding tradeoff. We then compare the speedup of $\mathrm{E}^{2} \mathrm{MC}$ with BDI and FPC and show the importance of decoding in parallel. 


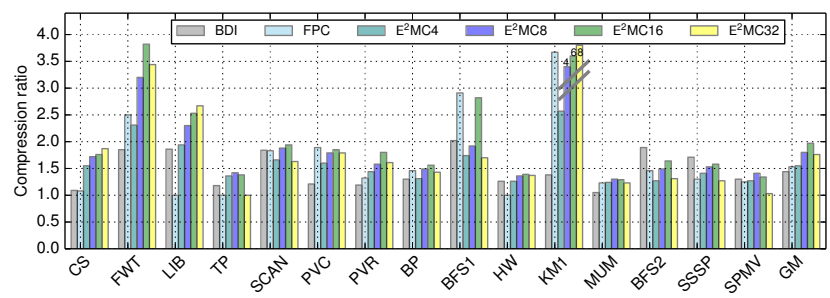

(a) Raw compression ratio

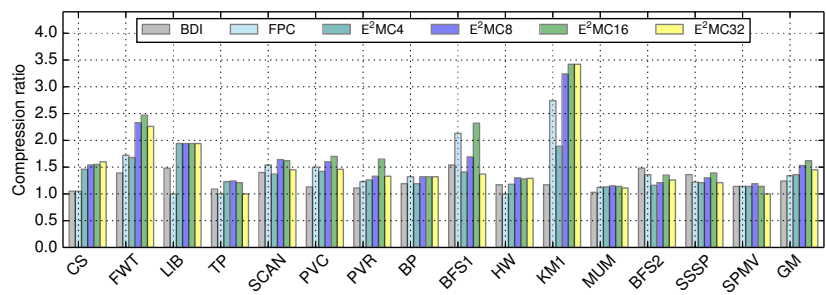

(b) Compression ratio at MAG

Fig. 9: Compression ratio of $\mathrm{BDI}, \mathrm{FPC}$ and $\mathrm{E}^{2} \mathrm{MC}$

Finally, we present the sensitivity analysis of compute-bound benchmarks to $\mathrm{E}^{2} \mathrm{MC}$ and study the energy efficiency of $\mathrm{E}^{2} \mathrm{MC}$.

\section{A. Compression Ratio using Offline Probability}

Figure 9a depicts the raw CR of BDI, FPC, and $\mathrm{E}^{2} \mathrm{MC}$ with offline probability. It shows that on average $\mathrm{E}^{2} \mathrm{MC}$ provides higher CR than BDI and FPC for all SLs. The geometric mean of the CR of $\mathrm{E}^{2} \mathrm{MC}$ for SLs 4, 8, 16 and 32-bit is $1.55 \times, 1.80 \times, 1.97 \times$, and $1.76 \times$, respectively, while that of BDI is only $1.44 \times$ and FPC is $1.53 \times$. This shows that entropy based compression techniques provide higher CR than simple compression techniques such as BDI and FPC whose CR is limited. $\mathrm{E}^{2} \mathrm{MC} 16$ yields the highest $\mathrm{CR}$ which is $53 \%$ and $42 \%$ higher than the CR of BDI and FPC respectively.

As discussed in Section III-C, data from memory is fetched in the multiple of MAG. Figure $9 \mathrm{~b}$ shows the CR of BDI, FPC and $\mathrm{E}^{2} \mathrm{MC}$ when this is taken into account. We see that the $\mathrm{CR}$ of all three techniques at MAG is less than the raw CR. However, the $\mathrm{CR}$ of $\mathrm{E}^{2} \mathrm{MC}$ is still higher compared to BDI and FPC. The geometric mean of the $\mathrm{CR}$ of $\mathrm{E}^{2} \mathrm{MC}$ for SLs $4,8,16$ and 32 -bit is $1.36 \times, 1.53 \times, 1.62 \times$, and $1.45 \times$, respectively, while the $\mathrm{CR}$ of $\mathrm{BDI}$ is only $1.24 \times$ and the $\mathrm{CR}$ of FPC is $1.34 \times$. We see that $\mathrm{E}^{2} \mathrm{MC} 16$ also yields the highest $\mathrm{CR}$ at MAG. So, we select $\mathrm{E}^{2} \mathrm{MC} 16$ and show CR results only for it and leave out other SLs for space reasons.

To obtain an estimate how close $\mathrm{E}^{2} \mathrm{MC} 16$ is to the optimal $\mathrm{CR}$, we calculate the upper bound on CR using Shannon's source coding theorem [25]. The average optimal CR for SL 16-bit is $2.61 \times$, which means there is a gap of $64 \%$ that could be further exploited. However, we think that further narrowing the gap is difficult as compression is data dependent.

1) Compression Ratio and Parallel Decoding Tradeoff: As shown in the Section III-B3, low decompression latency is important for achieving high performance. To reduce the decompression latency we decode in parallel as discussed in the Section III-F1. However, parallel decoding is not for free

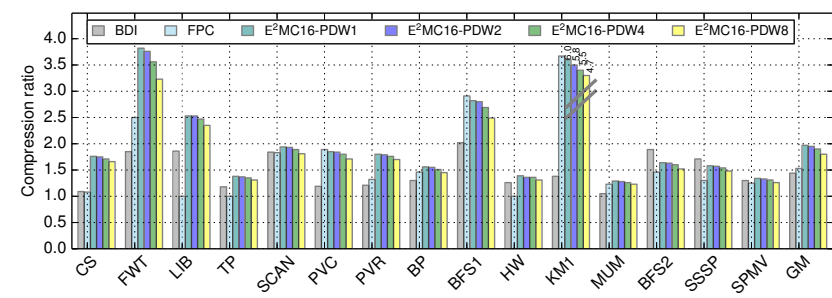

(a) Raw compression ratio

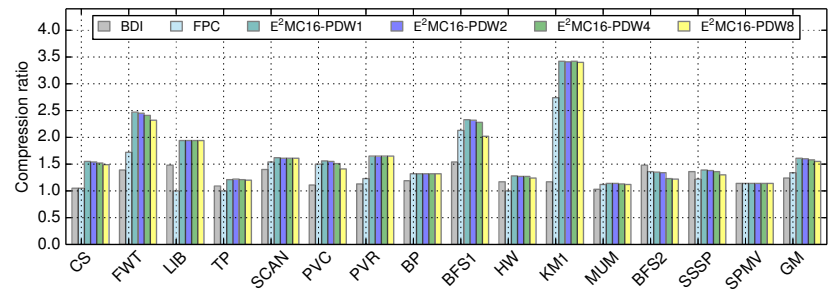

(b) Compression ratio at MAG

Fig. 10: Compression ratio of $\mathrm{E}^{2} \mathrm{MC} 16$ with parallel decoding

as it decreases the CR. Hence, the number of parallel decoding ways (PDWs) needs to be selected in a way such that the performance gain is maximal and the loss in CR is minimal.

Figure 10a shows that the CR decreases slightly when the number of PDWs increases. However, we will see in the Section V-C, the performance increases with the number of PDWs as the decompression latency decreases. Moreover, we see from the Figure 10a that the CR of $\mathrm{E}^{2} \mathrm{MC} 16$ even for 8 PDWs is still much higher than the CR of BDI and FPC.

We have seen that parallel decoding causes loss in CR. However, the loss in CR at MAG is much lower than the loss in raw CR as shown in Figure 10b than Figure 10a. For example, there is $9 \%$ loss in raw CR with 4 PDWs, while at MAG it is only $4 \%$. The reason for lower CR loss at MAG is that at MAG we usually need to fetch some extra bytes to meet the MAG requirements. Using these extra bytes to store offsets for parallel decoding does not cause loss in CR. This is not always true, however, and hence we see some loss in $\mathrm{CR}$ even at MAG.

\section{B. Compression Ratio using Online Sampling}

As discussed in the Section III-B2b online sampling might be needed to estimate probability if entropy characteristics are not known in advance. Figure 11a shows the CR for online sampling size of $20 \mathrm{M}$ instructions. We choose $20 \mathrm{M}$ instructions for online sampling as it gives the highest $\mathrm{CR}$ as shown in the Figure $4 \mathrm{~b}$ and we only sample at the beginning of each benchmark. The CR of $\mathrm{E}^{2} \mathrm{MC} 16$ is $1.79 \times$, which is $35 \%$ and $26 \%$ higher than the CR of BDI and FPC, respectively. However, as expected the CR with online sampling is lower by $18 \%$ on average than that of offline sampling.

Figure $11 \mathrm{~b}$ shows the $\mathrm{CR}$ with online sampling at MAG. The CR of $\mathrm{E}^{2} \mathrm{MC} 16$ at MAG is $1.52 \times$, which is still $28 \%$ and $18 \%$ higher than BDI and FPC, respectively. The CR results show that it is possible to achieve reasonably higher CR with 


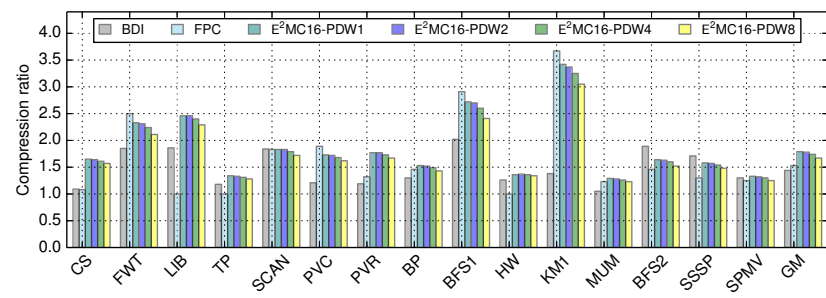

(a) Raw compression ratio

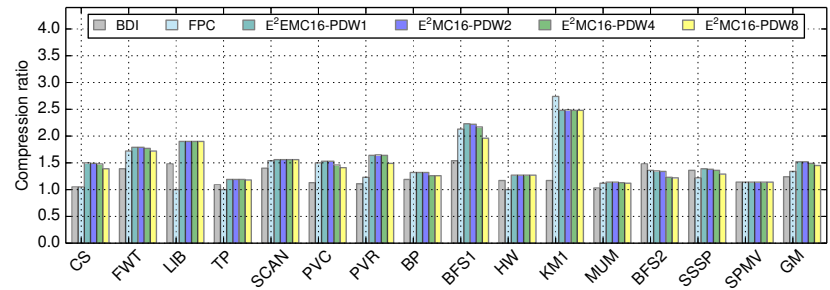

(b) Compression ratio at MAG

Fig. 11: Compression ratio of BDI, FPC and $\mathrm{E}^{2} \mathrm{MC} 16$ for online sampling size of $20 \mathrm{M}$ instructions

small online sampling. However, the CR at MAG with online sampling is $10 \%$ lower than offline sampling.

\section{Speedup}

We first establish an upper bound on the speedup assuming favorable conditions and then use realistic conditions to study the actual gain. Figure 12a shows the speedup of BDI, FPC, and $\mathrm{E}^{2} \mathrm{MC} 16$ when offline probability is used and the (de)compression latency is only one cycle for all techniques. BDI and FPC achieve an average speedup of $12 \%$ and $16 \%$, respectively, while the average speedup of $\mathrm{E}^{2} \mathrm{MC}$ is $16 \%, 21 \%$, $23 \%$, and $16 \%$ for SLs 4, 8, 16, and 32-bit, respectively. The speedup is due to the decrease in DRAM bandwidth which is reciprocal of the achieved compression ratio.

Figure 12b and Figure 12c shows the speedup of BDI, FPC and $\mathrm{E}^{2} \mathrm{MC} 16$ for offline and online sampling with realistic latencies as shown in the Table VI. For $\mathrm{E}^{2} \mathrm{MC}$ we only show speedup in detail for SL 16-bit with 1 to 8 PDWs because of the space reasons. A brief discussion of the speedup for SL 4, 8, and 32-bit is presented later in the section. We see that the speedup is less for FPC and $\mathrm{E}^{2} \mathrm{MC} 16$ using realistic latencies. However, the speedup of BDI does not change as actual latency is also single cycle. The speedup of $\mathrm{E}^{2} \mathrm{MC} 16$ with offline probability (13\%) is equal to the speedup of FPC (13\%) and even slightly less with online probability $(11 \%)$ when no parallel decoding is used, even though the $\mathrm{CR}$ of $\mathrm{E}^{2} \mathrm{MC} 16$ is much higher than that of FPC. This is because without parallel decoding the decompression latency of $\mathrm{E}^{2} \mathrm{MC} 16$ is 82 cycles, which is much higher than the decompression latency of FPC which is 10 cycles. The speedup increases when we increase the PDWs from 1 to 4 because each PDW decreases the decompression latency by half. However, each PDW also decreases the CR as we need to store the offsets for parallel decoding and hence there is a tradeoff between the CR and performance gain. Figure $12 \mathrm{~b}$ shows that there is no further

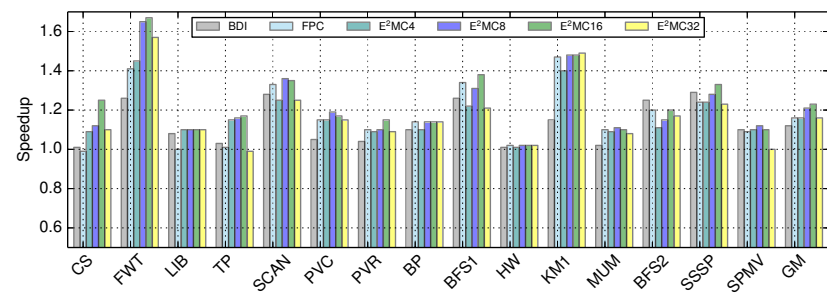

(a) Offline sampling with single cycle latency

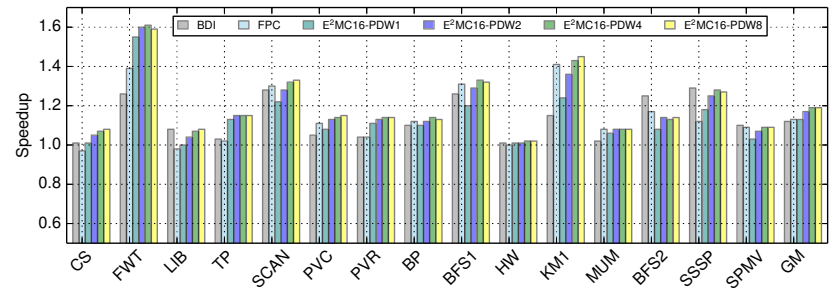

(b) Offline sampling with realistic latency

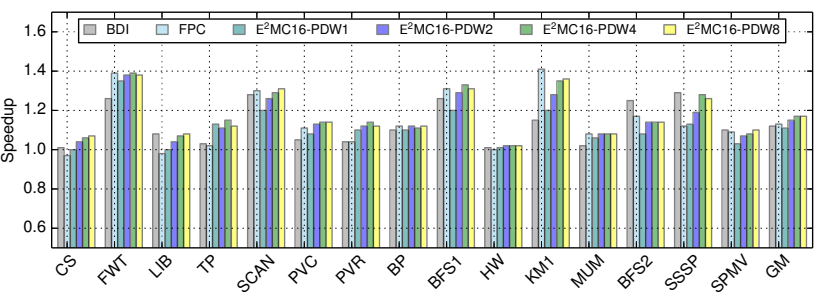

(c) Online sampling with realistic latency

Fig. 12: Speedup of BDI, FPC and $E^{2} M C$

gain in performance from 4 PDWs to 8 PDWs. This is because the increase in performance due to further decrease in latency is nullified by the decrease in CR. Hence, to achieve higher speedup for $\mathrm{E}^{2} \mathrm{MC} 16$ we not only need higher $\mathrm{CR}$ but also the decompression latency has to be reasonably low.

Figure 12a shows that the average speedup of $\mathrm{E}^{2} \mathrm{MC}$ for SL 8-bit with single cycle latency is much higher than the speedup of BDI and FPC and close to the speedup for SL 16-bit. The speedup for SLs 4 and 32-bit is also higher or equal to BDI and FPC. However, when actual latency is used the average speedup is much lower. The average speedup of $\mathrm{E}^{2} \mathrm{MC}$ for SLs 4, 8-bit with 8 PDWs and for SL 32-bit with 4 PDWs is $2 \%, 14 \%$ and $15 \%$ respectively. The reason for low speedup for SLs 4, and 8-bit is their high decompression latency.

We see that both offline and online sampling results in higher performance gain for $\mathrm{E}^{2} \mathrm{MC} 16$ than BDI and FPC, provided decompression latency is reduced by parallel decoding. The geometric mean of the speedup of $\mathrm{E}^{2} \mathrm{MC} 16$ with 4 PDWs is about $20 \%$ with offline probability and $17 \%$ with online probability. The average speedup is $8 \%$ higher than the state of the art with offline and 5\% higher with online sampling.

\section{Sensitivity Analysis of Compute-Bound Benchmarks}

We conduct sensitivity analysis to verify that $\mathrm{E}^{2} \mathrm{MC}$ increases performance of the memory-bound benchmarks without hurting the performance of the compute-bound benchmarks. The 


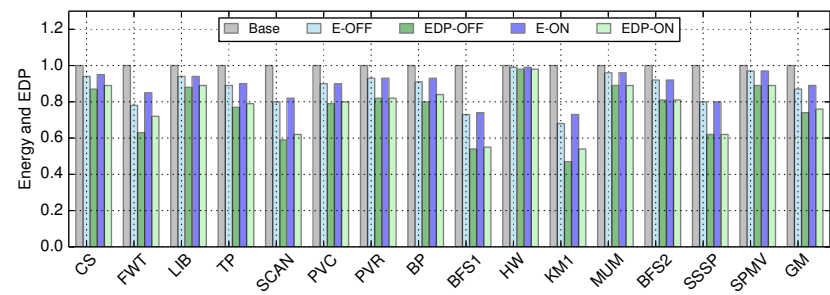

Fig. 13: Energy and EDP of $\mathrm{E}^{2} \mathrm{MC} 16$ for offline and online sampling over baseline

compute-bound benchmarks do not gain from increase in bandwidth and we compress them using $\mathrm{E}^{2} \mathrm{MC} 16$. On average there is only $1 \%$ reduction in performance for 1 PDW due to high decompression latency. However, we propose to use 4 PDWs and we notice no decrease in performance of the compute-bound benchmarks at 4 PDWs.

\section{E. Effect on Energy}

Figure 13 shows the reduction in energy consumption and energy-delay-product (EDP) over no compression for $\mathrm{E}^{2} \mathrm{MC} 16$ for offline and online sampling. On average there is $13 \%$ and $27 \%$ reduction in energy consumption (E-OFF) and EDP (EDP-OFF) respectively, for offline sampling and $11 \%$ and $24 \%$ reduction in energy consumption (E-ON) and EDP (EDP-ON) respectively, for online sampling. $\mathrm{E}^{2} \mathrm{MC} 16$ reduces the energy consumption by reducing the off-chip memory traffic and total execution time. We show energy and EDP of $\mathrm{E}^{2} \mathrm{MC}$ only over no compression due to lack of power models for BDI and FPC.

\section{CONCLUSIONS}

Most previous compression techniques were originally proposed for CPUs and hence, these techniques tradeoff low compression for low latency by using simple compression patterns. However, GPUs are less sensitive to latency than CPUs and they can tolerate increased latency to some extent. Thus, we studied the feasibility of relatively more complex entropy encoding based memory compression $\left(\mathrm{E}^{2} \mathrm{MC}\right)$ technique for GPUs which has higher compression potential, but also higher latency. We showed that $\mathrm{E}^{2} \mathrm{MC}$ is feasible for GPUs and delivers higher compression ratio and performance gain. We addressed the key challenges of probability estimation, choosing an appropriate symbol length, and decompression with reasonably low latency. $\mathrm{E}^{2} \mathrm{MC}$ with offline sampling results in 53\% higher compression ratio and $8 \%$ increase in speedup compared to the state-of-the-art techniques and saves $13 \%$ energy and 27\% EDP. Online sampling results in 35\% higher compression ratio and 5\% increase in speedup compared to the state of the art and saves 11\% energy and 24\% EDP. We also provided an estimate of the area and power needed to meet the high throughput of GPUs. In the future we plan to study the effect of multiple encodings and extend $\mathrm{E}^{2} \mathrm{MC}$ to other levels of the memory hierarchy.

\section{ACKNOWLEDGMENTS}

This work has received funding from the European Union's Horizon H2020 research and innovation programme under grant agreement No. 688759 (Project LPGPU2).

\section{REFERENCES}

[1] S. W. Keckler, W. J. Dally, B. Khailany, M. Garland, and D. Glasco, "GPUs and the Future of Parallel Computing," IEEE Micro, 2011.

[2] O. Mutlu, "Memory Scaling: A Systems Architecture Perspective," in Proc. 5th IEEE Int. Memory Workshop, IMW, 2013.

[3] "International Technology Roadmap for Semiconductors, ITRS," 2011.

[4] J. Leng, T. Hetherington, A. ElTantawy, S. Gilani, N. S. Kim, T. M. Aamodt, and V. J. Reddi, "GPUWattch: Enabling Energy Optimizations in GPGPUs," in Proc. 40th Int. Symp. on Comp. Arch., ISCA, 2013.

[5] J. Lucas, S. Lal, M. Andersch, M. A. Mesa, and B. Juurlink, "How a Single Chip Causes Massive Power Bills - GPUSimPow: A GPGPU Power Simulator," in Proc. IEEE Int. Symp. on Performance Analysis of Systems and Software, ISPASS, 2013.

[6] S. Lal, J. Lucas, M. Andersch, M. Alvarez-Mesa, A. Elhossini, and B. Juurlink, "GPGPU Workload Characteristics and Performance Analysis," in Proc. 14th Int. Conf. on Embedded Computer Systems: Architectures, Modeling, and Simulation, SAMOS, 2014.

[7] A. Alameldeen and D. Wood, "Frequent Pattern Compression: A Significance-Based Compression Scheme for L2 Caches," in Technical report, University of Wisconsin-Madison, 2004.

[8] X. Chen, L. Yang, R. Dick, L. Shang, and H. Lekatsas, "C-Pack: A High-Performance Microprocessor Cache Compression Algorithm," IEEE Transactions on VLSI Systems, 2010.

[9] G. Pekhimenko, V. Seshadri, O. Mutlu, P. B. Gibbons, M. A. Kozuch, and T. C. Mowry, "Base-Delta-Immediate Compression: Practical Data Compression for On-chip Caches," in Proc. 21st Int. Conf. on Parallel Architectures and Compilation Techniques, PACT, 2012.

[10] M. A. O'Neil and M. Burtscher, "Microarchitectural Performance Characterization of Irregular GPU Kernels," in Proc. IEEE Int. Symp. on Workloads Characterization, IISWC, 2014.

[11] ARM, "Frame Buffer Compression Protocol." [Online]. Available: https://www.arm.com/products/multimedia/mali-technologies/armframe-buffer-compression.php

[12] NVIDIA, "GPU Accelerated Texture Compression." [Online]. Available: https://developer.nvidia.com/gpu-accelerated-texture-compression

[13] V. Sathish, M. J. Schulte, and N. S. Kim, "Lossless and Lossy Memory I/O Link Compression for Improving Performance of GPGPU Workloads," in Proc. 21st Int. Conf. on Parallel Architectures and Compilation Techniques, PACT, 2012.

[14] N. Vijaykumar, G. Pekhimenko, A. Jog, A. Bhowmick, R. Ausavarungnirun, C. Das, M. Kandemir, T. Mowry, and O. Mutlu, "A Case for Core-Assisted Bottleneck Acceleration in GPUs: Enabling Flexible Data Compression With Assist Warps," in Proc. 42nd Int. Symp. on Comp. Arch., ISCA, 2015.

[15] S. Lee, K. Kim, G. Koo, H. Jeon, W. W. Ro, and M. Annavaram, "Warped-compression: Enabling Power Efficient GPUs Through Register Compression," in Proc. 42nd Int. Symp. on Comp. Arch., ISCA, 2015.

[16] A. Arelakis and P. Stenstrom, "SC2: A Statistical Compression Cache Scheme," in Proc. 41st Int. Symp. on Comp. Arch., ISCA, 2014.

[17] W. Fang, B. He, Q. Luo, and N. K. Govindaraju, "Mars: Accelerating MapReduce with Graphics Processors," IEEE Transactions on Parallel and Distributed Systems, 2011.

[18] M. Kulkarni, M. Burtscher, C. Cascaval, and K. Pingali, "Lonestar: A Suite of Parallel Irregular Programs," in Proc. Int. Symp. on Performance Analysis of Systems and Software, ISPASS, 2009.

[19] E. S. Schwartz and B. Kallick, "Generating a Canonical Prefix Encoding," ACM Comm., 1964.

[20] D. Salomon, "Variable-Length Codes for Data Compression," 2007.

[21] A. Bakhoda, G. Yuan, W. Fung, H. Wong, and T. Aamodt, "Analyzing CUDA Workloads Using a Detailed GPU Simulator," in Proc. IEEE Int. Symp. on Performance Analysis of Systems and Software, ISPASS, 2009.

[22] NVIDIA, "CUDA: Compute Unified Device Architecture," 2007, http://developer.nvidia.com/object/gpucomputing.html.

[23] S. Che, M. Boyer, J. Meng, D. Tarjan, J. Sheaffer, S.-H. Lee, and K. Skadron, "Rodinia: A Benchmark Suite for Heterogeneous Computing," in Proc. IEEE Int. Symp. on Workload Characterization, ISWC, 2009.

[24] A. Danalis, G. Marin, C. McCurdy, J. S. Meredith, P. C. Roth, K. Spafford, V. Tipparaju, and J. S. Vetter, "The Scalable Heterogeneous Computing (SHOC) Benchmark Suite," in Proc. 3rd Workshop on GPGPUs, 2010.

[25] C. E. Shannon, "A Mathematical Theory of Communication," $A C M$ SIGMOBILE Mobile Computing and Communications Review, 2001. 